\title{
Is there a correlation between the structure of hair and breast cancer or BRCA1/2 mutations?
}

\author{
Khalid Laaziri†, M. Sutton $\dagger$, P. Ghadirian $\ddagger$, A. Scott $\S$, A.-J. \\ Paradis§, P. N. Tonin§, and W. D. Foulkes \\ $\dagger$ Centre for the Physics of Materials and Department of Physics, McGill University, \\ Montréal, Québec, Canada H3A 2T8 \\ $\ddagger$ Epidemiology Research Unit, Centre Hospitalier d'Université de Montréal, \\ Hôtel-Dieu, 3849 rue St. Urbain, Montréal, Québec, Canada H3W-1T8 \\ $\S$ Research Institute of the McGill University Health Centre and the Departments of \\ Medicine and Human Genetics and Oncology, McGill University, 1650 Cedar avenue, \\ Room L10-116, Montréal, Québec, Canada H3G 1A4 \\ E-mail: mark@physics.mcgill.ca
}

\begin{abstract}
It has been suggested that the small angle $\mathrm{x}$-ray scattering (SAXS) pattern of human hair can be used to diagnose breast cancer and possibly to identify BRCA1/2 mutation carriers, who are at significantly elevated risk for developing breast cancer. In particular, the presence of a diffuse ring in the SAXS pattern was said to be diagnostic of either breast cancer or an increased risk thereof. To test this hypothesis, we measured SAXS from the pubic hair of 56 subjects with known BRCA1/2 and breast cancer status. We found that there is no clear association between the pattern of SAXS seen in human pubic hair and the risk of breast cancer or the presence of BRCA1/2 mutations. The possible use of SAXS to diagnose cancer remains conjectural, but this and previous studies do not suggest that SAXS can be used as a reliable method of identifying either BRCA1/2 mutation carriers or women who have had breast cancer.
\end{abstract}

Submitted to: Phys. Med. Biol.

PACS numbers: 


\section{Introduction}

Breast cancer is the most common cancer diagnosis in women living in North America (NIH 1996). Early detection of breast cancer can result in improved outcome, so any new test that might lead to an earlier diagnosis of breast cancer needs to be carefully evaluated. Recently, James and colleagues reported that the X-ray diffraction pattern from human hair could be used to diagnose breast cancer (James et al 1999). Even if this result does not lead to a reliable diagnostic technique, any demonstrable correlation between breast cancer and the structure of hair should to lead to new insights about breast cancer.

This intriguing hypothesis was soon followed by a rebuttal (Briki et al 1999), and several other publications documenting the failure to detect an association between hair structure and breast cancer risk (Amenitsch et al 1999, Schroder et al 1999, Chu et al 1999, Howell et al 2000 and Meyer et al 2000). One of the authors of the original study criticized these other reports for being unable to reproduce acceptable images from normal human hair (James 1999) and on the basis of a re-analysis of seven samples, convinced one group (Meyer et al 2000) to change their conclusion (Meyer and James 2001). We set out to improve upon previous methods by: (1) preparing the samples in accordance with instructions from the author of the original observation; (2) using the same experimental procedures and methodology as James stated (in fact, we have run our experiment simultaneously with the original author and following her protocols); (3) using a quantitative approach based on peak fitting, (4) using samples from women with a limited set of BRCA1/2 mutations (founder mutations observed in the French Canadian and Ashkenazi Jewish population); (5) using only pubic hair, so that effects of any head hair treatments would be avoided; (6) not using samples from patients who had received cancer chemotherapy in the previous 2 years. We report here our analysis of small angle x-ray scattering (SAXS) patterns from pubic hair samples. Since this result is still controversial, we think it is important to report the details of our analysis so others could duplicate the calculations.

\section{Materials and Methods}

After institutional review board approval, we wrote to 93 women who attended our hereditary cancer clinic or took part in research programs at McGill University and at the University of Montréal. Sixty-one samples of pubic hair were collected each with a signed informed consent form. Samples were analyzed anonymously in a double blind fashion. For reasons described below, fifty-six samples were usable. Table 1 summarizes the status of the participants, the line labeled no mutation represents participants who have no mutation detected in either BRCA1 or BRCA2. Twenty-four women have known BRCA1/2 mutations. Of these women, 19 have had breast cancer, and 5 have not been diagnosed with it. The thirty-two non-carriers included four women who are known not to have mutations that were present in the family, and twenty-one who do 
not have a family history of cancer and do not carry founder mutations that are common in the Ashkenazi Jewish and French Canadian populations. Sixteen of these twenty-one had been diagnosed with breast cancer for which they have been treated. Included in the group of non-carriers are seven healthy volunteers, two of whom are males. These also do not have a family history of cancer and were known not to have had breast cancer at the time of the sampling. These individuals have not been tested for BRCA1/2 mutations. All participants were sent an envelope with instructions regarding the study. Each person provided several pubic hairs for analysis. The samples were mailed to us in plastic quick-seal bags and stored at room temperature in Montréal before transfer to Chicago for analysis at the Advanced Photon Source.

The small angle x-ray scattering (SAXS) measurements were carried out on the Bio-CAT beamline at the Advanced Photon Source, using a focused beam of $\sim 180 \mu \mathrm{m} \times 35 \mu \mathrm{m}$ and a wavelength $\lambda=1.03 \AA$ (Irving et al 2000). The beam size was defined by a set of horizontal and vertical collimating slits. The setup includes one pair of guard slits just before the sample. The sample mount was placed in the air between two windows separating the downstream and upstream flight paths. The sample-to-detector distance was $R=932 \mathrm{~mm}$. The diffraction patterns were recorded on a Fuji Bas III imaging plates with an active area of $\sim 190 \mathrm{~mm} \times 240 \mathrm{~mm}$ so that the scattering was ranging from $\sim 0.0094 \AA^{-1}$ to $0.69 \AA^{-1}$. The exposure times were about 10 to 20 seconds. The data from the imaging plates were later extracted by electronic scans, each pixel has a $\Delta Q$ of $0.0013 \AA^{-1}$ (for scattering angle $2 \theta$ we define $Q=4 \pi \sin (\theta) / \lambda$ ). During the experiment single strands of hair were mounted on a cell, where they were gently stretched to ensure parallel and non-twisted alignment. The hair was then placed normal to the x-ray beam, and with the axis of the hair in the horizontal plane (labeled as the $\mathrm{x}$ direction in figure 1). The region between the sample and the detector was evacuated in order to minimize air scattering and absorption losses. This experimental setup is similar to one used in the original paper (James et al 1999) and produces similar high quality diffraction patterns meeting all criteria stressed by the main proponents of the technique (Meyer and James 2001, James 2001). For several subjects $(n=8)$ two different strands of hair were measured. Although not shown, the variation between these pairs is significantly smaller than the variation from sample to sample between subjects. Thus the observed variation from sample to sample is intrinsic to variations in the structure of hair between subjects.

\section{Results and discussion}

In order to analyse the diffraction pattern from hair, we have adopted two complementary approaches. The data were analyzed using a computer language called Yorick (Munro 1995). First, a qualitative analysis based on a visual presentation using a boxcar averaging routine in order to we arrive at images that are similar to those presented by James et al (1995). The basics of this routine is to remove the background scattering from the two-dimensional diffraction pattern using a smoothing 
procedure. The smoothing replaces the central pixel of a 19 by 19 box of pixels by its the average over the box. The smoothed image is then subtracted from the original image. Figure 1(a) shows a raw image from one hair sample and figure 1(b) shows the resulting processed image (the resolution of the spectrometer is much sharper than the features seen). Figure 1(b,c,d,e,f) shows the SAXS patterns (after processing) for several subjects. In agreement with previous investigations (Feughelman and James 1998 and James and Amemiya 1998), the diffraction patterns consists on a series of sharp and diffuse reflections (arcs) along the meridional direction, and spots along the equatorial. These spots arise from the hexagonal packing of the filaments across the hair. In the meridional direction the arcs arise from the long-range periodicity of $\alpha$-keratin filaments along the hair axis. The most intense and visible features of these reflections along the meridional are related to a $47.0 \mathrm{~nm}$ lattice, they are identified as the $7^{\text {th }}$ order peak $\left(Q=0.0935 \AA^{-1}\right)$, the $19^{\text {th }}$ order peak $\left(Q=0.254 \AA^{-1}\right)$, and the $38^{\text {th }}$ order peak ( $Q=0.508 \AA^{-1}$, outside the range of the plotted images). The $38^{t h}$ order peak of this lattice was used to accurately determine the center of the image and the sampleto-detector distance. In addition, when looking at the diffraction patterns in figure 1 , we can identify the presence of obvious diffuse rings in some images. In other images this scattering can be weak and it is hard to confirm their absence just by looking at the images. This is in part because the peak intensity and the background vary from hair sample to hair sample and one must carefully choose a colour scale as the exact intensity at which the colour changes can affect whether a small peak near that intensity level will appear in the image.

A quantitative analysis was developed in order to detect the presence or the absence of these rings. This procedure consisted of extracting one dimensional data from the SAXS images and peak identification of the resulting intensity curves by least squares fitting each peak to a Gaussian functional form. To extract the intensity curves, we circularly averaged the intensity of rectangular boxes (150 pixels along the radius and 10 pixels transversely) for two regions of the image starting from the center of the image along the meridional plane $0^{\circ}$, and along $45^{\circ}$ (see Fig.1f). A typical example is shown in figure 2. No other smoothing is applied to the data used for fitting. The intensity curve at $45^{\circ}$ was chosen to minimize the effect of the arcs of scattering in the meridional plane.

The $0^{\circ}$ data can be described as two broad peaks on a falling background. The $45^{\circ}$ data is similar except that often the low $Q$ peak is not there. This low $Q$ peak is the $7^{\text {th }}$ order arc of the $\alpha$-keratin structure which is a more extended for some samples.

The curve fitting procedure for the intensity curves consisted of fitting Gaussian peaks plus a quadratic background. The mathematical form used is

$$
l(Q)=I / \sqrt{2 \pi \sigma^{2}} \exp \left(-\left(Q-Q_{1}\right)^{2} /\left(2 \sigma^{2}\right)\right)
$$

which represents a peak at $Q_{1}$, with width $\sigma$ and integrated intensity $I$. During a first pass through the data, a set of 5 peaks was used for data from $0^{\circ}$ and a set of 3 peaks for the data from $45^{\circ}$. Equally good fits could be obtained by fixing the peaks positions and 
widths, so a second set of fits was performed where only the peak intensities (area under each peak) and the quadratic background were varied ( 8 and 6 parameters for the $0^{\circ}$ and the $45^{\circ}$ data respectively). Figure $2(\mathrm{a}, \mathrm{b})$ shows typical fitted intensity curves. No attempt was made to fully understand these peaks in terms of the underlying structure of hair. For the $0^{\circ}$ data the lower two peaks represent the $7^{\text {th }}$ order arc of the $\alpha$-keratin structure. This peak has a shoulder on the high $Q$ side. A fit of two Gaussian lineshapes positioned at $Q_{1}=0.0929 \AA^{-1}$ and $Q_{2}=0.0962 \AA^{-1}$ gives a good representation of its lineshape. This allows an accurate measure of the integrated intensity but clearly more work is needed to understand this lineshape. The higher $Q$ peak actually appears to be three peaks positioned at $Q_{3}=0.125 \AA^{-1}, Q_{4}=0.13 \AA^{-1}$ and $Q_{5}=0.14 \AA^{-1}$. The three peaks used to fit the $45^{\circ}$ data include the $7^{\text {th }}$ order arc which usually doesn't appear and two peaks that have the same peak positions as the two higher $Q$ peaks of the $0^{\circ}$ scattering. That the peak at $Q_{3}$ doesn't appear in the $45^{\circ}$ data suggests it is an arc of reflection arising from the $\alpha$-keratin structure. The corresponding d-spacings for these two diffuse peaks are $4.8 \mathrm{~nm}$ and $4.45 \mathrm{~nm}$ and they are believed to result from randomly oriented lipid granules that surround the $\alpha$-keratin filaments (Busson et al 1999). The relative intensity of these peaks varies from subject specimen to subject specimen as does the background levels. This method of analysis is a quantitative way to assess the intensity of peaks in the vicinity of the predicted cancer ring. The intensities given in this way correlate well with intensities assessed by a visual inspection of the diffraction images.

The integrated intensity of the two peaks $\left(Q_{4}\right.$ and $\left.Q_{5}\right)$ from the $45^{\circ}$ data sets are plotted in figure 3 separated by whether the subjects are affected or not affected by cancer, and figure 4 for subjects separated by whether they are carriers or non-carriers of BRCA1/2 mutations. The first pair $(a, b)$ in each figure are plots of the absolute integrated intensity. A second set of graphs $(\mathrm{c}, \mathrm{d})$ are presented in which the intensities are normalized using the integrated intensity of the $7^{\text {th }}$ order peak (sum of $Q_{1}$ and $Q_{2}$ intensities) in the corresponding $0^{\circ}$ data. This intensity scales as the amount of hair in the beam and partially corrects for the different diameter hairs. It is evident that the scatter of the intensities is much reduced in plots $(\mathrm{c}, \mathrm{d})$. An alternate normalization, the background intensity from 0.11-0.115 $\AA^{-1}$ was also used and this is shown in graphs $(\mathrm{e}, \mathrm{f})$. This also reduces the scatter in the intensities although not as much as using the $7^{\text {th }}$ order peak intensity.

It is apparent from these data that the ring is present in almost all samples at varying levels of intensity. In addition, a plot of the intensities of either the $Q_{4}$ or $Q_{5}$ peaks versus participant's age show no correlation. During the analysis some of the samples $(n=4)$ were rejected as their diffuse rings had a very high intensity, and Meyer et al assigned this effect to another cause (Meyer and James 2001). Another participant was rejected because we were unable to get a clean diffraction pattern even after using several pieces of her hair. These five rejected samples are why the sample size was reduced from 61 to 56 .

The original report concluded that the presence of breast cancer lead to the 
appearance of a ring of scattering in the 23 people with breast cancer and the lack of a diffuse ring was seen in 24 of 28 people without breast cancer. To test for a similar effect in our data, an attempt was made to determine a threshold intensity level. For the several thresholds tried, a chi-square test gives results consistent with random probabilities. Clearly, any simple analysis based on the presence or absence of a diffuse peak is inconsistent with our data and the high level of correlation found in the original report (James et al 1999). To test for a possible difference in average intensity, a Student's t test can be used. Tables 2 and 3 present the results of such tests for the data sets presented in figure 3 and figure 4 . The three rows of table 2 shows t-tests for each method of intensity normalization of the $Q_{4}$ ring. Column one of the table tests for any intensity difference between subjects with and without breast cancer. Column two tests for any difference between subjects with any BRCA mutation and those with none. Table 3 presents similar information based on the $Q_{5}$ ring intensities. The probabilities obtained in all cases indicate that the differences in the means are not statistically significant. Although this sample size is not large enough to rule out a small correlation between intensity and either effect, it does rule out the high level of correlation implied in the original study.

A limitation of this study is that it tested hair samples collected from women well after their treatment for breast cancer. The structure of hair changing due to the cancer treatments could disguise any effect. However, one would expect that if the original hypothesis was correct, the occurrence of a diffuse peak should be suppressed and this is not observed. An experiment designed to use samples from people who have been diagnosed but not yet treated would eliminate this possible effect. Note that since BRCA1/2 mutation status will not change with time or treatment, any such effect will not change our findingw with respect to mutation status. From our results it is clear there is no correlation with the intensity of the diffuse ring and BRCA1/2 mutations. It is also clear that a significant fraction of the non-cancer population can have the presence of a diffuse ring in the SAXS patterns of their hair. Thus, there is no clear correlation between either a person's BRCA1/2 mutation status or their breast cancer status and the presence of a ring of scattering as found by James et al (1999).

\section{Conclusion}

Previous studies (Briki et al 1999, Amenitsch et al 1999, Schroder et al 1999, Chu et al 1999, Howell et al 2000 and Meyer et al 2000) have also been unable to replicate the findings of James et al (1999). Considerable controversy was generated by the original report and the subsequent publications. The latest publication (James 2001) reaches the opposite conclusion from a remeasurement of the same samples in Meyer et al (2000). Clearly, it is important to try and replicate these experiments. Furthermore, a more quantitative method should be used to determine the presence or absence of the suggested ring of scattering. The quantitative method described above was applied to 56 samples and no evidence for an association between an x-ray diffraction ring and either 
the subject's BRCA1/2 status or the breast cancer status could be found, and this is incompatible with James et al (1999). Taken together with the previous publications, we have to conclude that SAXS from a human hair cannot be used as a simple diagnostic for breast cancer. Nevertheless, there is an association between certain rare disorders and abnormal hair structure (Dawber and Blackwell 1997); and for these diseases further well controlled studies to determine differences in x-ray scattering patterns may be warranted. It is also important to emphasize the variability seen in this region of the SAXS from our 56 samples, and it is clear much work remains to be done in order to understand the origin of these features in the SAXS from human hair.

\section{Acknowledgments}

We would like to thank Tom Irving for his help in using the beamline at Bio-CAT, and Chantal Perret for assistance with the sample collection. This work was funded by grant from the Canadian Breast Cancer Research Initiative administered by the National Cancer Institute of Canada. Use of the APS was supported by the U.S. DOE (BES, OER) under Contract No. W-31-109-Eng-38.

\section{References}

Amenitsch H, Rappolt M, Laggner P, Bernstorff S, Moslinger R, Fleishmann E, T Wagner, S Lax, E Petru, K Hudabiunigg, and L Dalla Palma 1999 Synchrotron X-ray study at Trieste: No correlation between breast cancer and hair structure Synchrotron and Radiation News 12 32-4

Briki F, Busson B, Salicru B, Esteve F, and Doucet J, 1999 Breast-cancer diagnosis using hair Nature 400226

Busson B, Engström P, and Doucet J, 1999 J Synchrotron Rad 6 1021-10

Chu B, Fang D, and Hsiao BS 1999 Hair test results at the Advanced Polymers Beamline (X27C) at the NSLS, Synchrotron and Radiation News 1236

Dawber E, 1997 Diseases of the hair and scalp (Blackwell Science).

Feughelman M, and James V 1998 Hexagonal Packing of Intermediate Filaments (Microfibrils) in $\alpha$-keratin Fibers Text Res $J \mathbf{6 8}$ 110-4

Howell A, Grossmann JG, Cheung KC, Kanbi L, Evans DG, and Hasnain SS 2000 Can hair be used to screen for breast cancer? J Med Genet 37 297-8

Irving T C, Fischetti R, Rosenbaum G, and Bunker G B 2000 Fiber diffraction using the BioCAT Undulator beamline at the Advanced Photon Source Nucl. Inst \& Meth A 448 250-5

James V 1999 Comments on the statements and experiments contained in this review Synchrotron and radiation News 12 31-2

James V, 2001 The importance of good images in using hair to screen for breast cancer $J$ Mol Genet 38 e16

James V and Amemiya Y 1998 Intermediate Filaments in $\alpha$-keratin of Echidna Quill Text Res J 68 $167-70$

James V, Kearsley J, Irving T, Amemiya Y, and Cookson D 1999 Using hair to screen for breast cancer Nature 398 33-4

Meyer P, Goergl R, Botz JW, and Fratzl P 2000 Breast cancer screening using small-angle X-ray scattering analysis of human hair $J$ Natl Cancer Inst 92 1092-3

Meyer P and James V 2001 Experimental confirmation of a distinctive diffraction pattern in hair from women with breast cancer $J$ Natl Cancer Inst 93 873-5 
Munro DH 1995 Using the Yorick interpreted language Comp Phys9 609-15

NIH Cancer rates and risks, (Bethesda: NIH), 1996

Schroder K, Abumrad N, Derisi D, Kastrow K, Busch E, Volkow E, and Capel M 1999 Attempts to replicate the claim of James's at the NSLS beam line X12B Synchrotron and Radiation News 12 $34-5$

Wilk KE, James VJ, and Amemiya Y 1995 The intermediate filament structure of human hair Biochim Biophys Acta $1245392-6$ 
Table 1. Status of participants in study.

\begin{tabular}{lrrr}
\hline & $\begin{array}{c}\text { Breast } \\
\text { cancer }\end{array}$ & $\begin{array}{r}\text { No breast } \\
\text { cancer }\end{array}$ & Subtotals \\
\hline BRCA1 mutation & 9 & 0 & 9 \\
BRCA2 mutation & 9 & 5 & 14 \\
Both mutations & 1 & 0 & 1 \\
No mutation & 16 & 16 & 32 \\
\hline Subtotals & 35 & 21 & 56 \\
\hline
\end{tabular}

Table 2. Student's t test and probabilities for the $Q_{4}$ ring. There are 35 samples from people who have had breast cancer and 21 from those who have not. Also, there are 24 samples from people with BRCA1/2 mutations and 32 from those without.

\begin{tabular}{lll}
\hline & Breast cancer & BRCA1/2 \\
\hline Absolute & $\mathrm{t}=0.89$ & $\mathrm{t}=0.18$ \\
& $\mathrm{P}=0.38$ & $\mathrm{P}=0.86$ \\
\hline Normalized & $\mathrm{t}=1.03$ & $\mathrm{t}=0.19$ \\
(7th order) & $\mathrm{P}=0.31$ & $\mathrm{P}=0.85$ \\
\hline Normalized & $\mathrm{t}=0.69$ & $\mathrm{t}=0.24$ \\
(background) & $\mathrm{P}=0.50$ & $\mathrm{P}=0.81$ \\
\hline
\end{tabular}

Table 3. Student's t test and probabilities for the $Q_{5}$ ring. Sample sizes are the same as table 2 .

$$
\text { Breast cancer BRCA1/2 }
$$

\begin{tabular}{lll}
\hline Absolute & $\mathrm{t}=0.14$ & $\mathrm{t}=0.23$ \\
& $\mathrm{P}=0.89$ & $\mathrm{P}=0.82$ \\
\hline Normalized & $\mathrm{t}=0.59$ & $\mathrm{t}=0.65$ \\
( ${ }^{\text {th }}$ order $)$ & $\mathrm{P}=0.55$ & $\mathrm{P}=0.52$ \\
\hline Normalized & $\mathrm{t}=0.43$ & $\mathrm{t}=0.11$ \\
(background) & $\mathrm{P}=0.67$ & $\mathrm{P}=0.91$ \\
\hline
\end{tabular}




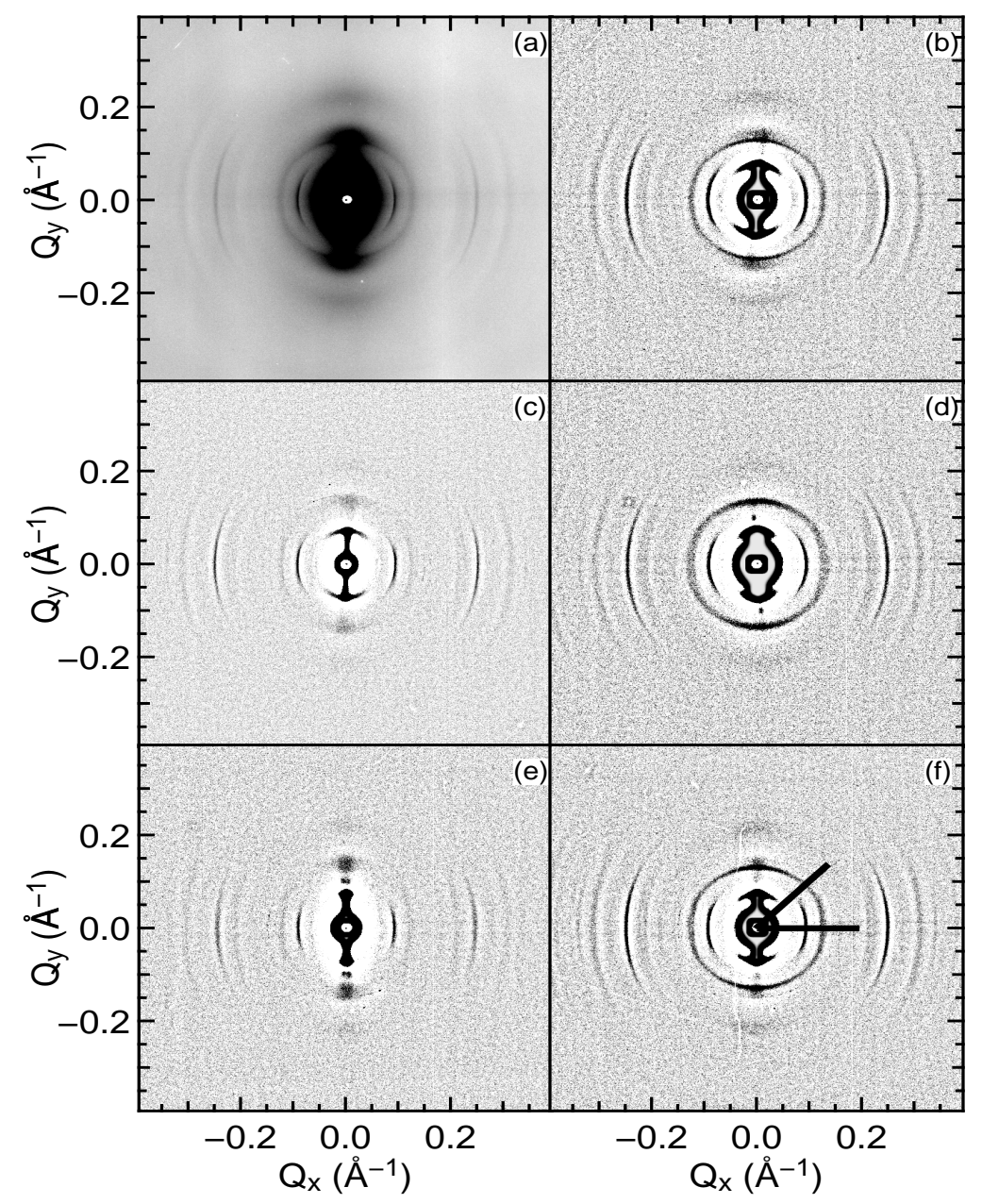

Figure 1. Diffraction patterns from human hair: (a) raw image, (b) same image after treatment (subtracting a smoothed image), (c) and (d) treated images for patient diagnosed with breast cancer, (e) and (f) images from patient not diagnosed with breast cancer. The hair axis is along $Q_{x}$. The 2 black strips on (f) at $0^{\circ}$ and $45^{\circ}$ represents the two pixel regions averaged over in order to do the Gaussian fitting. 

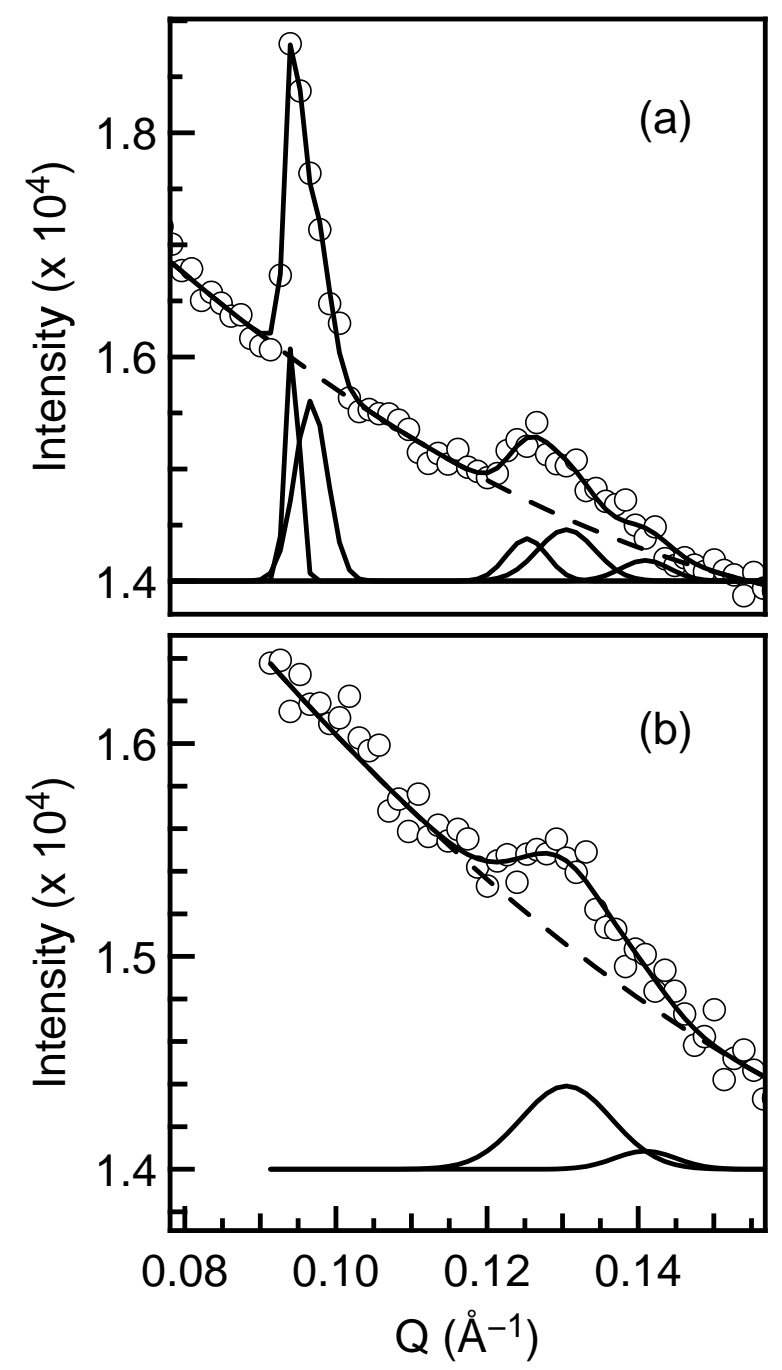

Figure 2. Circularly averaged data (circles) with fitted curves described in the text (solid lines through data points). The decomposition of the curve into it's component parts is also shown. The dashed line is the fitted background curve and the Gaussian peaks are offset to a base line of $1.4 \times 10^{4}$ for clarity. This sample is from a patient who was affected by breast cancer and has a BRCA1 mutation. (a) Along $0^{\circ}$. (b) Along $45^{\circ}$. For this sample only two Gaussian peaks are needed. 


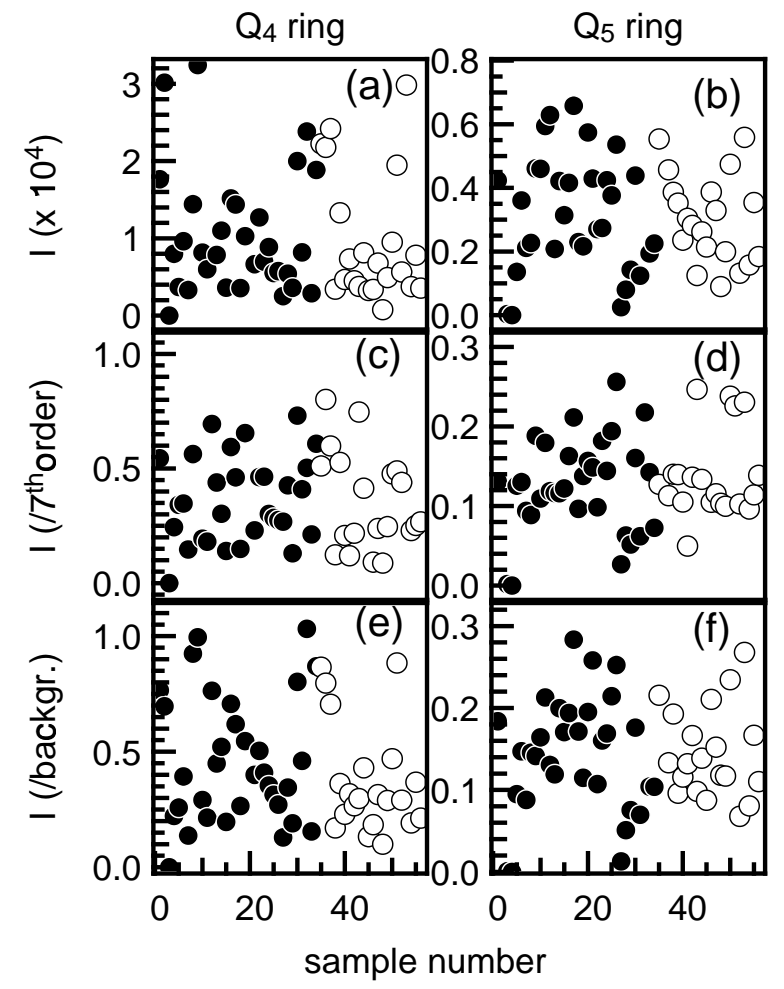

Figure 3. Integrated intensities of the two peaks $\left(Q_{4}\right.$ ring and $Q_{5}$ ring) in the case of patients affected (filled symbols) or not by cancer (open symbols). Filled and open squares are for patients with a BRCA1/2 mutation and filled and open circles are for patients without. (a) Absolute intensity of $Q_{4}$ ring, (b) Absolute intensity of $Q_{5}$ ring, (c) Normalized intensity of $Q_{4}$ ring using the $7^{\text {th }}$ order peak intensity, (d) Normalized intensity of $Q_{5}$ ring using the $7^{\text {th }}$ order peak intensity, (e) Normalized intensity of $Q_{4}$ ring using background, and (f) Normalized intensity of $Q_{5}$ ring using background. For reference, the $Q_{4}$ ring in figure 2(b) has integrated intensities 5400, 0.43 and 0.35 for the three ways of normalizing the data (figure $3 \mathrm{a}, 3 \mathrm{c}$ and $3 \mathrm{e}$ respectively) and the $Q_{5}$ ring has values $800,0.06$ and 0.05 for the same normalization methods. 


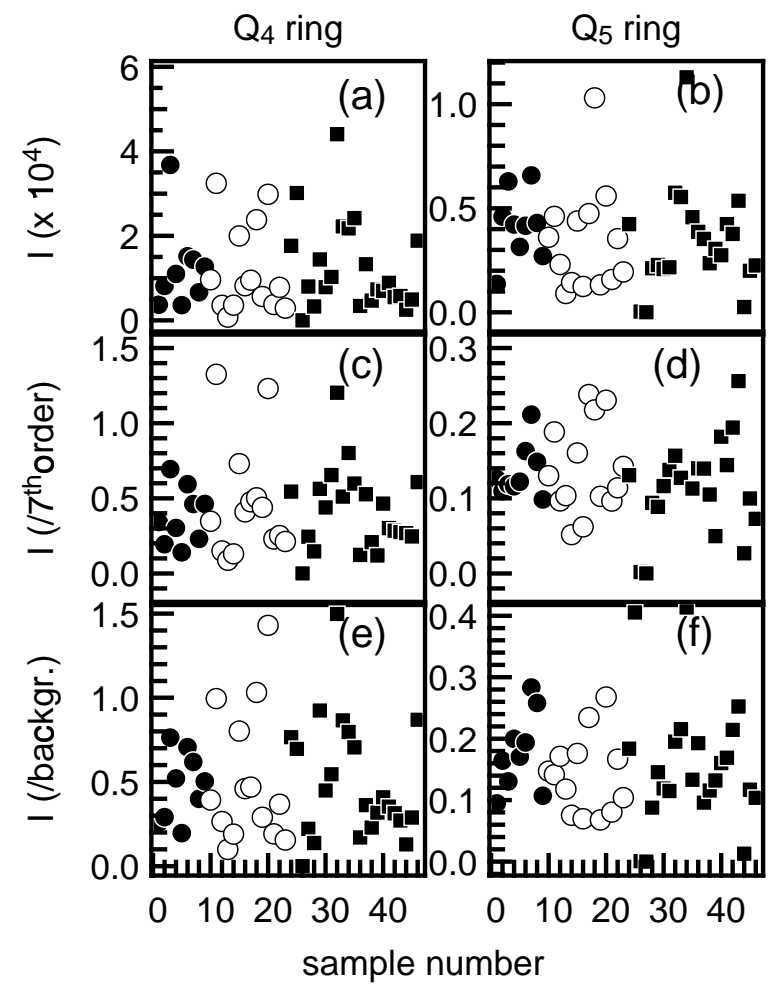

Figure 4. Integrated intensity of the two peaks $\left(Q_{4}\right.$ ring and $Q_{5}$ ring) separated into those with BRCA1 mutations (filled circles), BRCA2 mutations (open circles) and those without (filled squares): (a) Absolute intensity of $Q_{4}$ ring, (b) Absolute intensity of $Q_{5}$ ring, (c) Normalized intensity of $Q_{4}$ ring using the $7^{t h}$ order peak intensity, (d) Normalized intensity of $Q_{5}$ ring using the $7^{\text {th }}$ order peak intensity, (e) Normalized intensity of $Q_{4}$ ring using background, and (f) Normalized intensity of $Q_{5}$ ring using background. 